\title{
Mössbauer and Magnetic Properties of Coherently Mixed Magnetite-Cobalt Ferrite Grown by Infrared Pulsed-Laser Deposition
}

Juan de la Figuera,,${ }^{1, *}$ Adrián Quesada, ${ }^{2}$ Laura Martín-García, ${ }^{1}$ Mikel Sanz,${ }^{1}$ Mohamed Oujja, ${ }^{1}$ Marta Castillejo, ${ }^{1}$ Arantzazu Mascaraque, ${ }^{3}$ Alpha T. N'Diaye, ${ }^{4}$ Michael Foerster, ${ }^{5}$ Lucía Aballe, ${ }^{5}$ José F. Marco ${ }^{1, *}$

\author{
1 Instituto de Química Física "Rocasolano", CSIC, Madrid E-28006, Spain \\ 2 Instituto de Cerámica y Vidrio, CSIC, Madrid E-28049, Spain \\ 3 Universidad Complutense de Madrid, Madrid E-28040, Spain \\ ${ }^{4}$ Lawrence Berkeley National Laboratory, Berkeley, California 94720, USA \\ ${ }^{5}$ ALBA Synchrotron Light Facility, CELLS, Barcelona ES-08290, Spain \\ * Corresponding author's e-mail address: juan.delafiguera@iqfr.csic.es, jfmarco@iqfr.csic.es
}

RECEIVED: October 1, 2015 * REVISED: December 3, 2015 * ACCEPTED: December 4, 2015

THIS PAPER IS DEDICATED TO DR. SVETOZAR MuSIĆ ON THE OCCASION OF HIS 70

\begin{abstract}
We have studied the magnetic properties and the composition of cobalt ferrite single crystal films on $\mathrm{SrTiO}_{3}$ : Nb grown by infrared pulsed-laser deposition. Mössbauer spectra have been recorded from both the target used to grow the films and the films themselves. The Mössbauer spectra of the target taken at low temperatures show a strong dependence of the recoil free fraction of the octahedral sites with temperature. The films composition, with a coexistence of Co-enriched cobalt ferrite and magnetite, has been estimated assuming a similar ratio of the recoil free fractions of the films. X-ray absorption and $x$-ray magnetic circular dichroism measurements confirm the valence composition of the film and show ferromagnetic Fe-Co coupling in the films with a coercive field around $0.5 \mathrm{~T}$ at room temperature. The combination of these characterization techniques allows establishing the coherent structural and magnetic properties of this biphase system.
\end{abstract}

Keywords: cobalt ferrite, oxide spinels, pulsed laser deposition, Mössbauer spectroscopy.

\section{INTRODUCTION}

$\mathbf{M}$ IXED iron-cobalt spinel oxides have attracted interest for magnetic applications for a long time. One reason is that the ternary Co-Fe oxide spinel comprises a series that extends from the archetypical magnetic material, magnetite $\left(\mathrm{Fe}_{3} \mathrm{O}_{4}\right)$, to the antiferromagnetic $\mathrm{CO}_{3} \mathrm{O}_{4}$ spinel and includes solid solution phases of intermediate compositions, opening the range to tune the magnetic and electronic properties to suit particular applications. For example one particular composition, Co : Fe $1: 2$-cobalt ferrite proper-corresponds to the ferrite with the highest magnetocrystalline anysotropy. ${ }^{[1]}$ In this particular case the origin of such high magnetic anisotropy lies in the high orbital moment of the $\mathrm{Co}^{2+}$ cations in octahedral sites. The cation distribution of iron-cobalt spinels also depends on the Co : Fe ratio. While magnetite is an inverse spinel, i.e. the divalent $\mathrm{Fe}$ cations are located only in the octahedral sites, $\mathrm{Co}_{3} \mathrm{O}_{4}$ is a direct spinel with all the $\mathrm{Co}^{2+}$ in the tretrahedral sites. Cobalt ferrite is mostly an inverse spinel structure, although some $\mathrm{Co}^{2+}$ can be found at tetrahedral sites. Both magnetite and cobalt ferrite present a ferrimagnetic order with the $A$ and $B$ lattices coupled antiferromagnetically, with a magnetic moment per formula unit which is higher for magnetite $(4.0 \mu \mathrm{B})$ than for cobalt ferrite $(3.4 \mu \mathrm{B})$. The decrease in the latter is due to the cancellation of the contributions of the high-spin $\mathrm{Fe}^{3+}$ cations in octahedral and tetrahedral sites.

Adjusting the ratio of $\mathrm{Co}$ and Fe has been achieved in molecular beam epitaxy growth by changing the Fe: Co ratio, ${ }^{[2,3]}$ with the films being presumably homogeneous. We note that it is complicated to detect a segregated 
Fe-Co oxide with averaging techniques, or even resolved ones, if both phases are coherent. In this work, we used pulsed laser deposition ${ }^{[4]}$ with wavelengths in the infrared range to make the films. This wavelength is more unusual that the typical ultraviolet range. ${ }^{[5-8]}$ In this work, we will show how Mössbauer spectroscopy is ideally suited to detect the mixture of magnetite and cobalt ferrite that is obtained under reducing conditions (i.e. high vacuum environment) on $\mathrm{SrTiO}_{3}: \mathrm{Nb}(100)$ substrates. Such substrate is a popular one for the growth of spinel oxides because its conductivity can be controlled by $\mathrm{Nb}$ doping, and the (100) orientation promotes a simple cube-on-cube epitaxial relationship with a mostly relaxed ferrite lattice parameter. ${ }^{[9]}$

\section{EXPERIMENTAL}

The cobalt ferrite targets were synthesized by a sol-gel process. It involved Fe and Co nitrates, $\mathrm{Co}\left(\mathrm{NO}_{3}\right)_{2} \times 6 \mathrm{H}_{2} \mathrm{O}$ and $\mathrm{Fe}\left(\mathrm{NO}_{3}\right)_{2} \times 9 \mathrm{H}_{2} \mathrm{O}$, which were dissolved in the appropriate $1: 2$ ratio in deionized water. Citric acid was added to the solution, and stirred at $333 \mathrm{~K}$ for $1 \mathrm{~h}$.

Afterwards, the gel was dried for $24 \mathrm{~h}$, and calcined for $1 \mathrm{~h}$ at $673 \mathrm{~K}$. The powders were then sieved $\leq 63 \mu \mathrm{m}$ and pressed at $55 \mathrm{MPa}$ into disks of $15 \mathrm{~mm}$ in diameter and $2 \mathrm{~mm}$ in thickness. At a final stage, the targets were sintered at $1473 \mathrm{~K}$ for $2 \mathrm{~h}$ in air.

Cobalt ferrite films were grown by pulsed-laser deposition (PLD) on $\mathrm{SrTiO}_{3}$ : $\mathrm{Nb}$ (100) (0.1\%) substrates (Crystek) with a $1064 \mathrm{~nm}$ wavelength Q-switched Nd:YAG laser using $15 \mathrm{~ns}$ pulses, a $10 \mathrm{~Hz}$ repetition rate, a fluence of $4 \mathrm{~J} \mathrm{~cm}^{-2}$ and a total deposition time of 4 hours. The conditions employed are similar to our previous study of the growth of magnetite. ${ }^{[9,10]}$ The substrates, in high vacuum, were heated to $748 \mathrm{~K}$ during deposition.

The crystalline structure was studied by XRD (Philips XPert) using $\mathrm{Cu} K_{\alpha}(0.154184 \mathrm{~nm})$ radiation in the $\vartheta / 2 \vartheta$ configuration. ${ }^{57} \mathrm{Fe}$ Mössbauer spectra were recorded in constant acceleration mode with a ${ }^{57} \mathrm{Co} / \mathrm{Rh}$ source in conventional constant acceleration spectrometers.

Integral Conversion Electron Mössbauer Spectroscopy (ICEMS) data for the films was acquired at RT using a parallel plate avalanche counter.[11] Target spectra were measured in the transmission mode in a helium closedcycle cryorefrigerator. ${ }^{[12]}$ The spectra were computer-fitted and theisomer shift data are quoted relative to metallic iron at room temperature.

The X-ray absorption and circular dichroism experiments were performed at the Magnetic Spectroscopy 6.3.1 beamline of the Advanced Light Source (ALS) at Berkeley National Laboratory (USA) and the CIRCE beamline of the ALBA Synchrotron Light Facility (Spain). In the former, x-ray absorption spectroscopy (XAS) and x-ray magnetic circular dichroism (XMCD) spectra were taken at the magnetic spectroscopy and mapping beamline 6.3.1 of the Advanced Light Source in total electron yield mode. A magnetic field of \pm 1.9 T was applied parallel to the $X$-ray beam which had a fixed circular polarization of $\approx 60 \%$. The sample surface normal was tilted by $60^{\circ}$ with respect to the beam and the field. Each XMCD spectrum was acquired within $7 \mathrm{~min}$. At the CIRCE beamline, spectro-microscopy was performed in remanence with a spectroscopic and low-energy electron microscope (SPELEEM). ${ }^{[13,14]}$ The microscope can be used either for low-energy electron microscopy and diffraction (LEEM ${ }^{[15]}$ and LEED) or with X-rays, i.e. in photoelectron microscopy mode (XPEEM). In XPEEM mode it can acquire images of the energy-filtered photoelectrons with a spatial resolution of $20 \mathrm{~nm}$. Using a pass energy of $2 \mathrm{eV}$ provides an spatially resolved distribution of electrons that is similar in depth sensitivity to the total electron yield mode in the ALS setup. In order to obtain XMCD images, images acquired with opposite $x$-ray helicity are substracted pixelby-pixel. The $\mathrm{X}$-ray beam impinges on the sample at $16^{\circ}$ from the surface plane, and the magnetic contrast is detected along the $\mathrm{X}$-ray direction.

\section{RESULTS AND DISCUSSION}

In order to characterize properly the composition of the thin films we first describe the target characterization, mostly done by Mössbauer spectroscopy, with special emphasis on the dependence of the recoil-free fraction of the octahedral sites with temperature. We then proceed to further characterize the films by $x$-ray absorption spectroscopy (XAS) and $\mathrm{x}$-ray magnetic circular dichroism (XMCD).

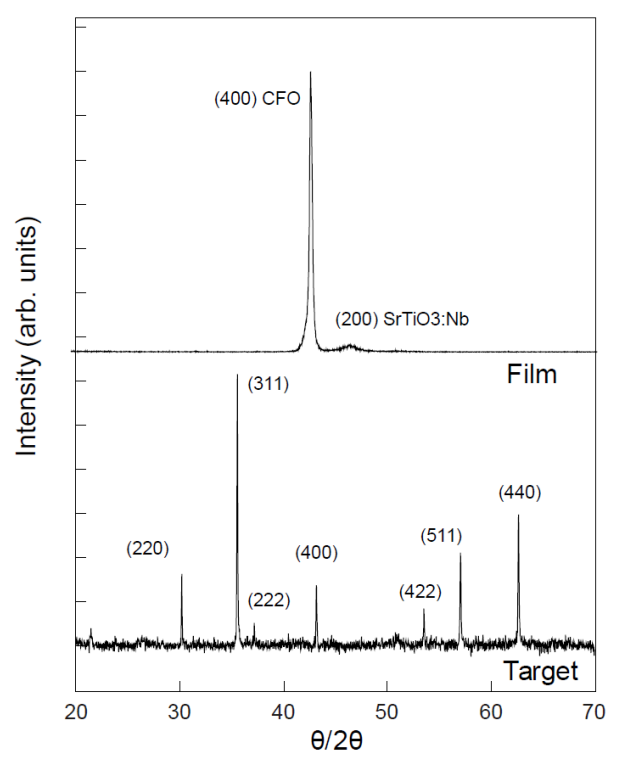

Figure 1. X-ray diffraction in a $\vartheta / 2 \vartheta$ configuration. Bottom: target, Top: film. CFO: cobalt ferrite. 


\section{Target Characterization}

The cobalt ferrite target has been characterized by $\mathrm{x}$-ray diffraction (XRD) and Mössbauer spectroscopy. The XRD shows only spinel polycrystalline peaks, with the (220), (311), (222), (400), (422), (511) and (440) reflections detected shown in Figure 1.

The Mössbauer spectra are shown in Figure 2. At room temperature (RT), a clear magnetic pattern is observed with no singlet or doublet contribution. Similar spectra have been previously reported for $\mathrm{CoFe}_{2} \mathrm{O}_{4},{ }^{[16-19]}$ confirming that cobalt ferrite is magnetically ordered at room temperature. The highest velocity peak, at $7 \mathrm{~mm} \mathrm{~s}^{-1}$, shows a clear assymetry that strongly suggests that at least it involves two different components. For stoichiometric cobalt ferrite $-\left(\mathrm{Fe}^{3+}\right)_{A}\left[\mathrm{Co}^{2+} \mathrm{Fe}^{3+}\right]_{B} \mathrm{O}^{4-}$, where all the iron is in a $3+$ state and the cobalt in the $2+$ state, two closely spaced sextets are expected corresponding to $\mathrm{Fe}^{3+}$ in either octahedral and tetrahedral sites with a ratio between the two components of $1: 1$. In consequence the spectrum has been fitted to two sextet contributions, whose parameters are shown in Table 1. In the RT spectrum, the isomer shift $(\delta)$,

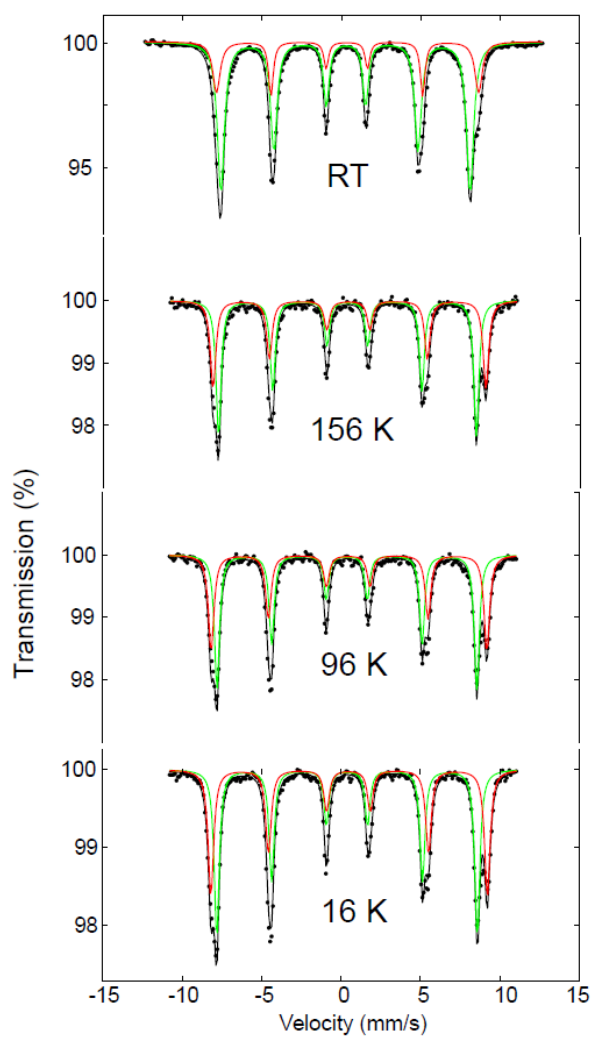

Figure 2. Mössbauer spectrum of a cobalt ferrite target at different temperatures. Two sextets components, corresponding to $\mathrm{Fe}^{3+}$ in tetrahedral (green) and octahedral (red) sites respectively, have been used for the fit. The fit is shown by a black line. quadrupole shift $(2 \varepsilon)$ and hyperfine fields correspond clearly to $\mathrm{Fe}_{\text {oct }}^{3+}\left(0.36 \mathrm{~mm} \mathrm{~s}^{-1}, 0.04 \mathrm{~mm} \mathrm{~s}^{-1}\right.$ and $51.2 \mathrm{~T}$ respectively) and $\mathrm{Fe}_{\text {tet }}^{3+}\left(0.27 \mathrm{~mm} \mathrm{~s}^{-1},-0.02 \mathrm{~mm} \mathrm{~s}^{-1}\right.$, and $48.7 \mathrm{~T}$ respectively), the areas instead correspond to a much different $A / B$ ratio of 2.9 .

Such large difference between each component area is striking. There are two possibilities to explain it: either (i) the recoil free fraction of iron cations in the two sites is very different at room temperature, or (ii) the recoil free fraction is similar but the relative population of octahedral and tetrahedral iron cations is very different from the expected $1: 1$ one. In fact, it is known that cobalt ferrite is often only partially inverse, with up to $30 \%$ of extra iron in the octahedral site. ${ }^{[3,20]}$ Thus, changes in the relative populations of octahedral and tetrahedral iron would be expected when heating cobalt ferrite above RT as cation diffusion is activated (typically at temperatures above $370 \mathrm{~K}$ ). In contrast, when cooling below RT no such change of population should be observed as cationic diffusion is expected to be frozen. In order to check this dependence, the Mössbauer spectra have been measured at decreasing temperatures of $196 \mathrm{~K}, 96 \mathrm{~K}$ and $16 \mathrm{~K}$, shown in figure 2 . As observed in the spectra, the two components become better separated at lower temperature, due mostly to the change in the hyperfine fields that increase from the RT value of 48.7 T for the tetrahedral sites to $50.9 \mathrm{~T}$, while the octahedral site hyperfine field increases from 51.2 to 53.9 T. But more obvious, the relative areas of the two sextets used in the fits change from the RT $A / B$ ratio of 2.9 to 1.2 at $16 \mathrm{~K}$.

Table 1. Mössbauer parameters used for the fit in the temperature dependent transmission spectra of the target. $\delta$ - isomer shift, in $\mathrm{mm} \mathrm{s}^{-1} ; 2 \varepsilon$ - quadrupole shift, in $\mathrm{mm} \mathrm{s}^{-1}$; $H$ - hyperfine magnetic field in Tesla; Area- percentage of the signal in each component.

\begin{tabular}{ccccc}
\hline Temperature $/ \mathrm{K}$ & $\delta$ & $2 \varepsilon$ & $H$ & Area \\
\hline 300 & & & & \\
$\mathrm{Fe}_{A}^{3+}$ & 0.27 & -0.02 & 48.7 & 74.5 \\
$\mathrm{Fe}_{B}^{3+}$ & 0.36 & 0.04 & 51.2 & 25.5 \\
157 & & & & \\
$\mathrm{Fe}_{A}^{3+}$ & 0.35 & 0.00 & 50.3 & 59.0 \\
$\mathrm{Fe}_{B}^{3+}$ & 0.46 & 0.08 & 53.3 & 41.0 \\
96 & & & & \\
$\mathrm{Fe}_{A}^{3+}$ & 0.37 & 0.00 & 50.7 & 54.0 \\
$\mathrm{Fe}_{B}^{3+}$ & 0.48 & 0.06 & 53.8 & 46.0 \\
16 & & & & \\
$\mathrm{Fe}_{A}^{3+}$ & 0.38 & 0.00 & 50.9 & 54.7 \\
$\mathrm{Fe}_{B}^{3+}$ & 0.49 & 0.04 & 53.9 & 45.3 \\
\hline
\end{tabular}


The area of each component is directly proportional to the number of atoms in a given environment and the recoil free fraction for the corresponding nuclei. In turn, the recoil free fraction $f$ is a function of the mean quadratic displacement of each atom, ${ }^{[21]} f=\exp \left(-E_{\mathrm{R}}^{2}\left\langle x^{2}\right\rangle / \hbar c^{2}\right)$. A common way to model the dependence of the mean quadratic displacement is through the Debye model, which applied to the recoil free fraction gives:

$$
f=\exp \left[-\frac{3 E_{R}}{2 k_{B} \Theta}\left(1+\frac{4 T^{2}}{\Theta^{2}} \int_{0}^{\Theta / T} \frac{x d x}{e^{x}-1}\right)\right]
$$

where $E_{\mathrm{R}}$ is the recoil energy of ${ }^{57} \mathrm{Fe}$ for the $14.4 \mathrm{keV}$ gamma ray, and $\Theta$ corresponds to the Debye temperature, or more correctly, to the characteristic Mössbauer temperature for a particular nucleus.

One way to visualize the evolution of the recoil free fraction of iron in each of the cation sites is to plot the ratio of the recoil free fraction, estimated by the Debye model, as a function of temperature for different Debye temperatures for $\mathrm{Fe}_{\text {tet }}^{3+}$ and $\mathrm{Fe}_{\text {oct }}^{3+}$. Such ratio is shown in Figure 3a for several values of the respective Debye temperatures, as well as the experimental data. This strong dependence of the recoil free fraction for cobalt ferrite has been previously reported by Kim et al. ${ }^{[16,22]}$ It was interpreted as a characteristic Debye temperature of $734 \mathrm{~K}$ for $\mathrm{Fe}_{\text {tet }}^{3+}$ and $248 \mathrm{~K}$ for $\mathrm{Fe}_{\text {oct }}^{3+}$. In our case, the lower temperature data points are reasonably reproduced with those Debye temperatures, although the $R T$ ratio requires an even larger difference of Debye temperatures. It is clear that a large difference in the Debye temperature of octahedral and tetrahedral sites is required to account for the difference in recoil free fraction at room temperature.

Another way to estimate the Debye temperatures is through the second order Doppler (SOD) shift ${ }^{[21]}$ of the isomer shift. In this case, a temperature dependent isomer shift is assumed to arise from the mean square velocity of each atom, $\delta_{\text {SOD }}=-E_{R}\left\langle v^{2}\right\rangle / 2 c^{2}$. Again within the Debye model, the mean square velocity can be written in terms of the Debye temperature:

$$
\delta_{\mathrm{SOD}}=\delta_{1}-\frac{9 k_{\mathrm{B}} T}{2 M c}\left(\frac{T}{\Theta}\right)^{3} \int_{0}^{\Theta / T} \frac{x^{3} d x}{e^{x}-1}
$$

As shown in Figure $3 \mathrm{~b}$ the experimental data, although following the trend in temperature, do not fall on the predicted curves for either Kim et al. ${ }^{[16]}$ Debye temperatures, or the more appropriate ones that correspond to our room temperature data of (735 K / $190 \mathrm{~K})$. A more detailed study would be interesting to understand the (a)

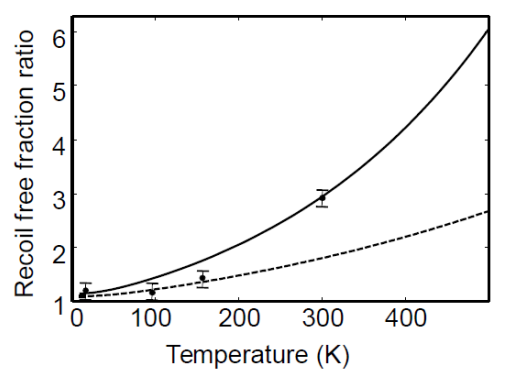

(b)

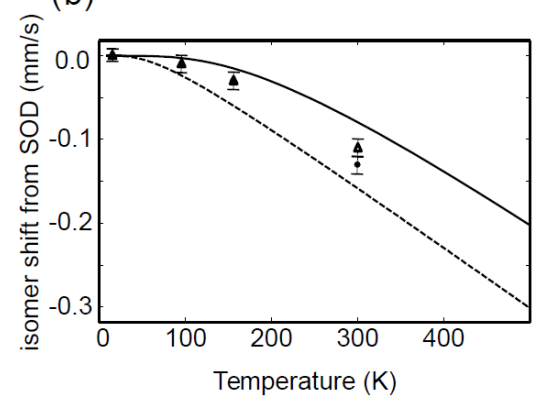

Figure 3. (a) Plot of the ratio of recoil free fraction vs sample temperature. The continuous curve corresponds to $\Theta_{A}=$ $735 \mathrm{~K}$ and $\Theta_{B}=190 \mathrm{~K}$, while the dashed one corresponds to $\Theta_{A}=735 \mathrm{~K}$, and $\Theta_{B}=250 \mathrm{~K}$, and the experimental points are marked with circles. (b) Isomer shift expected from the second order Doppler shift. The continuous lines correspond to the Debye model with characteristic Debye temperature of 250 (dashed line), and $735 \mathrm{~K}$ (continuous line). The experimental change in isomer shifts with temperature of the $A$ sites are shown by triangles, while the ones of the B sites are shown by filled circles.

source of this lack of agreement, although it is worth mentioning that both estimates are unequally affected by the high-frequency lattice vibrations cut off in the Debye model. ${ }^{[21]}$ The discrepancy might also be due to the strong overlap of the two sextets at $R T$ which precludes a rigorous determination of their respective spectral areas as mentioned in Ref. 21.

The main conclusion from our study is that the signal from $\mathrm{Fe}_{\text {oct }}^{3+}$ is strongly reduced when the temperature is increased from low temperature to $R T$, although it is not necessary to go below liquid nitrogen temperatures to obtain a reliable measure of the recoil free fraction of cobalt ferrite. The strong temperature dependence of the $\mathrm{Fe}_{\text {oct }}^{3+}$ recoil free fraction remains, so far, a puzzling result without theoretical explanation.

\section{Films Characterization}

The XRD pattern of one of the films, shown in Figure 1, has only two reflections, one from the substrate and a second one corresponding to the spinel (400) reflection from the 
film. This confirms the film single crystal nature, as suggested by other structural techniques. ${ }^{[23]}$

Two Mössbauer spectra from batches of films grown under nominally similar conditions are shown in Figure 4. The contrast with the cobalt ferrite target is quite evident. Unlike the small asymmetry observed in the high velocity side arising from the two iron cations with the same $3+$ oxidation state and two environments, in the films there is a clear shoulder on the lower velocity peaks. Taking advantage of the recoil free fraction of each iron cation with $3+$ valence in the cobalt ferrite target, we have fitted the films spectra to two sextets with the same isomer and quadrupole shifts and hyperfine fields used in the cobalt ferrite target plus an additional sextet (see Table 2). The additional sextet has parameters that correspond closely to those shown by the octahedral component of magnetite, which has a mixed valence of $2.5+$ due to electron hopping between the $\mathrm{Fe}^{2+}$ and $\mathrm{Fe}^{3+}$ during the Mössbauer process ( $\delta$ of $0.67 \mathrm{~mm} \mathrm{~s}^{-1}$ and $H=46.5 \mathrm{~T}$ ). Thus, the most direct interpretation is that the films are composed of a coherent mixture of magnetite and cobalt ferrite (there is an additional doublet which is attributed to a small fraction of $\mathrm{FeO})$.

The amount of magnetite and cobalt ferrite can be calculated from the Mössbauer spectra if some simplifying assumptions are made. First, we assume that the magnetite

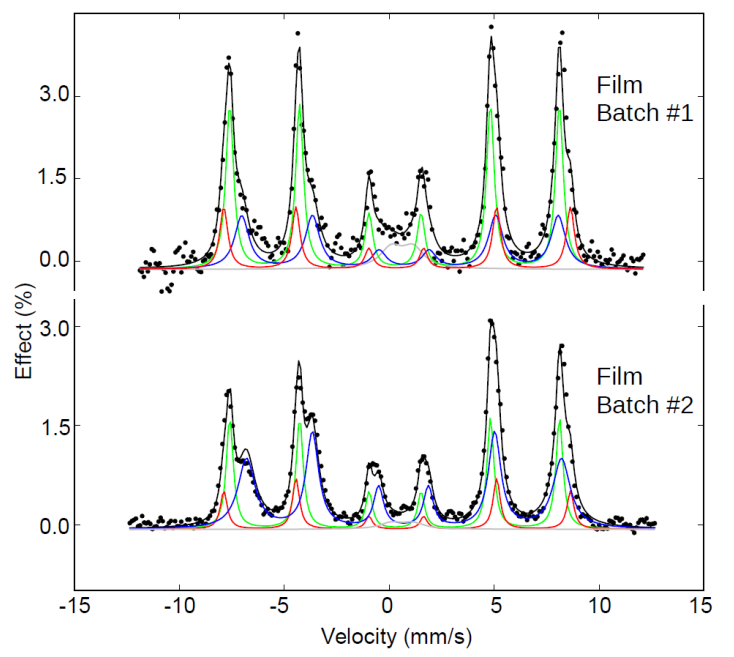

Figure 4. Integral Conversion Electron Mössbauer spectra of different series of films of cobalt ferrite on $\mathrm{SrTiO}_{3}$ grown under nominally identical conditions. Each component is shown in color. In addition to the sextex components already used in the target fits (which correspond respectively to $\mathrm{Fe}^{3+}$ in octahedral sites (red) and $\mathrm{Fe}^{3+}$ is tetrahedral ones (green)), two components have been used: a sextet that corresponds to $\mathrm{Fe}^{2.5+}$ in octahedral sites (blue) and a double which corresponds to FeO (gray). The fit is shown by a black line. component is stoichiometric and that the cobalt ferrite one is a spinel (fixing the ratio of tetrahedral and octahedral cations to $1: 2$ ), but allowing for both iron and cobalt cations in each environment (i.e., octahedral or tetrahedral site) with a possible oxygen deficiency. We also fix the total amount of $\mathrm{Co}:$ Fe to $1: 2$.

In order to obtain the relative populations of the two phases we need to know the relative recoil free fractions of the different cations involved. For magnetite, we use the ratio for tetrahedral and octahedral iron (1.9) which has been interpreted as arising from the very similar characteristic Debye temperatures of $334 \mathrm{~K}$ and $314 \mathrm{~K}$ by Vandenberghe et al. in Ref. 21. The ratios between the recoil free fraction of the $\mathrm{Fe}_{\text {tet }}^{3+}$ components of cobalt ferrite and magnetite is assumed to be 1.3 as it arises from the respective Debye temperatures of $725 \mathrm{~K}^{[16]}$ and $334 \mathrm{~K} .{ }^{[21]}$ Using all the constraints together provides a composition of each film and also the detailed cation distribution and oxygen deficiency of the cobalt ferrite phase, which given some of the assumptions should be taken as a rough indication of the relative importance of the magnetite component in the film rather than as a definite statement of each component composition. We note that, due to their isostructural nature, most material science characterization techniques will be relatively blind to this kind of coherent mixture of magnetite and cobalt ferrite, highlighting the need for Mössbauer spectroscopy characterization in this context. The results for the first batch of films is $68 \%$ of $\left(\mathrm{Fe}^{3+}{ }_{0.8} \mathrm{Co}^{2+}{ }_{0.2}\right)\left[\mathrm{Co}^{2+}{ }_{1.2} \mathrm{Fe}^{3+}{ }_{0.8}\right] \mathrm{O}_{3.8}, 22 \%$ of $\mathrm{Fe}_{3} \mathrm{O}_{4}$, and $9 \% \mathrm{FeO}$, and for the second is $52 \%$ of $\left(\mathrm{Fe}^{3+}{ }_{0.7} \mathrm{Co}^{2+}{ }_{0.3}\right)\left[\mathrm{Co}^{2+}{ }_{1.5} \mathrm{Fe}^{3+}{ }_{0.5}\right] \mathrm{O}_{3.6}$, $44 \%$ of $\mathrm{Fe}_{3} \mathrm{O}_{4}$, and $3 \% \mathrm{FeO}$.

Table 2. Mössbauer parameters used for the fit in the temperature dependent transmission spectra of the target. $\delta$ - isomer shift, in $\mathrm{mm} \mathrm{s}^{-1} ; 2 \varepsilon$ - quadrupole shift (for sextets) or $\Delta$ - quadrupole splitting (for doublets), both in $\mathrm{mm} \mathrm{s}^{-1}$; $H$ - hyperfine magnetic field in Tesla; Area- percentage of the signal in each component.

\begin{tabular}{ccccc}
\hline & $\mathrm{IS}$ & $2 \varepsilon, \Delta$ & $H$ & Area \\
\hline Film batch \#1 & & & & \\
$\mathrm{Fe}_{A}^{3+}$ & 0.27 & -0.02 & 48.7 & 48.9 \\
$\mathrm{Fe}_{B}^{3+}$ & 0.36 & 0.04 & 51.2 & 14.1 \\
$\mathrm{Fe}_{B}^{2.5+}$ & 0.61 & -0.18 & 46.7 & 30.2 \\
$\mathrm{FeO}$ & 0.61 & 1.05 & - & 6.8 \\
$\mathrm{Film} \mathrm{batch} \mathrm{\# 2}^{3+}$ & & & \\
$\mathrm{Fe}_{A}^{3+}$ & 0.27 & -0.02 & 49.2 & 41.0 \\
$\mathrm{Fe}_{B}^{3+}$ & 0.36 & 0.04 & 51.3 & 5.6 \\
$\mathrm{Fe}_{B}^{2.5+}$ & 0.67 & -0.02 & 46.5 & 51.3 \\
$\mathrm{FeO}^{3+}$ & 0.61 & 0.77 & - & 2.1 \\
\hline
\end{tabular}


The likely physical origin of the presence of magnetite lies in the reducing conditions of the high vacuum chamber during the pulsed laser deposition growth. In fact, in our previous work we studied the reduction from an hematite target to magnetite films grown under the same conditions. ${ }^{[9,10]}$ This seems to imply that the conditions are such that Fe cannot stay solely in a $\mathrm{Fe}^{3+}$ oxidation state. Once some $\mathrm{Fe}^{2+}$ is deposited on the surface, the film prefers to form a dual phase of magnetite and cobalt-enriched cobalt ferrite. This in turn allows to form a coherent (as the XRD pattern suggests) film with two different phases. As this would be a novel method of tuning the properties of mixed $\mathrm{Fe}-\mathrm{Co}$ ferrites, we proceed to consider the magnetic properties of some of these films. The structural properties of the second batch of samples, with $40 \%$ magnetite content, have been studied in a separate work. ${ }^{[23]}$

In the two batches of films, the ratio of the Mössbauer peaks is not compatible with a random orientation of the magnetization. Rather, the $3: 3: 1$ ratio of the peak areas suggests that the magnetization has an out-of-plane preference. In order to learn more about the magnetic properties of the films, we have performed $x$-ray absorption and $x$-ray magnetic circular dichroism on the first batch of samples (\#1, which have a fraction of $22 \%$ magnetite). The $\mathrm{x}$-ray absorption spectra of the $\mathrm{L}_{2-3}$ edges as measured in remanence in PEEM are presented in Figure $5 \mathrm{a}$ and $\mathrm{b}$. Similar spectra were measured in by total yield XAS (not shown). The Fe XAS spectra shows the typical structure characteristic of a film containing mostly $\mathrm{Fe}^{3+}$ cations. ${ }^{[2]}$ Likewise, the Co spectra is typical of $\mathrm{Co}^{2+}$ cations. ${ }^{[2]}$ While we have assumed that Fe to be $3+$, the Mössbauer spectra for this sample suggests that it has up to $20 \%$ magnetite, which corresponds to $7 \%$ of $\mathrm{Fe}^{2+}$.

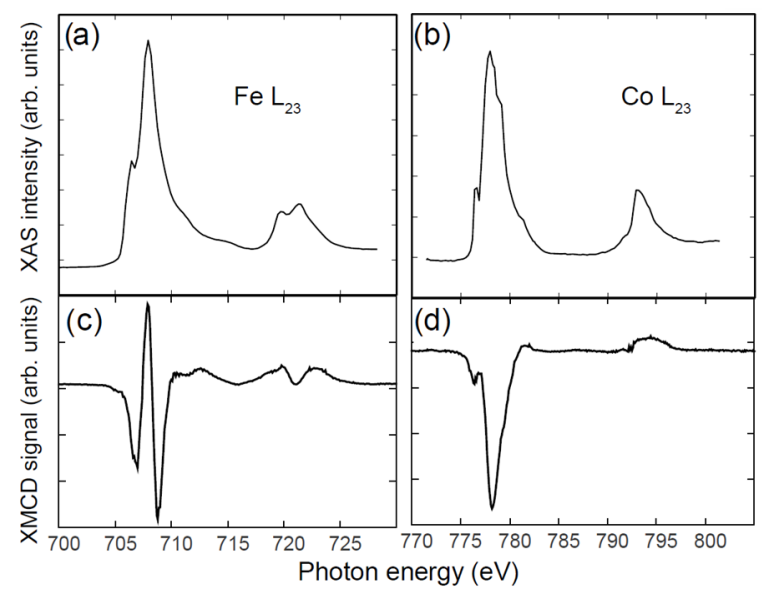

Figure 5. (a) and (b) X-ray absorption spectra of the L edges of Fe and Co respectively, measured in PEEM; (c) and (d), XMCD spectra of the same L edges.
XMCD spectra measured at room temperature under a 1.9 $\mathrm{T}$ magnetic field are shown in Figure $5 \mathrm{c}$ and $\mathrm{d}$ for the $\mathrm{Fe}$ and $\mathrm{Co}_{\mathrm{L}_{2-3}}$ edges respectively. For the $\mathrm{Fe}_{3}$ edge, a three peak structure is observed in Figure $5 c$, with two negative and one positive incursions. This structure arises from iron in several environments and nominal valences, as reported for ferrite XMCD studies. ${ }^{[24]}$ In a naive approach each of the peaks of the Fe edge XMCD spectra corresponds respectively to $\mathrm{Fe}^{2+}$ in octahedral sites, $\mathrm{Fe}^{3+}$ in tetrahedral sites and $\mathrm{Fe}^{3+}$ in octahedral sites in order of increasing photon energy. Nevertheless, this is a simplification as measurements in maghemite, which only has $\mathrm{Fe}^{3+}$ cations, also show a three peak structure, ${ }^{[24]}$ albeit with a smaller first downward peak. As described before, the Mössbauer spectra suggested that about $20 \%$ magnetite should be present. This is in reasonable agreement with the XMCD spectra of Figure $5 c$, where the first downward peak is smaller that expected for pure magnetite, ${ }^{[24]}$ but larger than either in $\mathrm{CoFe}_{2} \mathrm{O}_{4}{ }^{[3]}$ or maghemite, ${ }^{[24]}$ which both only have $\mathrm{Fe}$ in a $3+$ oxidation state. The XMCD Co $\mathrm{L}_{3}$ edge, like the XAS spectra, suggests the presence only of $\mathrm{CO}^{2+}$ cations. The sense of the XMCD Co spectrum indicates that the octahedral cobalt and the iron cations magnetic moments are oriented in the same direction.

In Figure 6a the hysteresis cycles of Fe and Co are measured by plotting the amplitude of the XMCD signal in total electron yield at photon energies corresponding to the largest (downward) peaks of the $\mathrm{L}_{3} \mathrm{XMCD}$ spectra of Fe and Co. We note that the sample magnetization is not completely saturated even at a field of 1.9 T. The different hysteresis cycles, when normalized by the maximum of the XMCD intensity at the corresponding energy, fall on top on one another, suggesting that the iron and cobalt cations magnetic moments are strongly coupled to each other. The comparison of the hysteresis cycle at room temperature and at $100 \mathrm{~K}$ are shown in Figure $6 \mathrm{~b}$. At lower temperatures, the width of the hysteresis cycle increases, reflecting an increased magnetocrystalline anisotropy. The coercive field is $\sim 0.5 \mathrm{~T}$ at room temperature. The lack of two different components in the hysteresis loops at low temperature suggests a strong coupling between the magnetite and the cobalt ferrite components. Furthermore, the minority magnetite component has not decreased radically the coercive field of the complete film. It is also likely that the film has a substantial number of antiphase boundaries that pin down the magnetic domains. Applying the sum rules to the Fe and Co absorption edges provides a measurement of the orbital and spin moments of each element in the film. ${ }^{[25]}$ To such end, the number of $d$-holes in each cation is required (the ratio of spin and orbital moment is independent of such estimate). To get an estimate, we assumed that all the iron is in $\mathrm{Fe}^{3+}$ form, while all $\mathrm{Co}$ is in $\mathrm{Co}^{2+}$ form. In such case, the estimated magnetic moment per Fe cation is 
$m_{\mathrm{s}}=1.05 \mu \mathrm{B}$ and $m_{\mathrm{o}}=0.1 \mu \mathrm{B}$ for the spin and the orbital components, respectively. In contrast, the Co cation has $m_{\mathrm{s}}=1.53 \mu \mathrm{B}$ and $m_{\mathrm{o}}=0.65 \mu \mathrm{B}$. The relative numbers point to a larger contribution (per atom) of the orbital moment of cobalt, as expected from its $2+$ oxidation state. In contrast $\mathrm{Fe}$, mostly in a $3+$ oxidation state, has negligible orbital moment.

We have imaged the film surface with the photoemission microscope, detecting the secondary electron emission as a proxy of the X-ray absorption. ${ }^{[26]}$ An image taken at the $\mathrm{Co}_{\mathrm{L}} \mathrm{L}_{3}$ edge is shown in Figure 7. Weak contrast variations are observed in the image close to the resolution limit of the microscope. Magnetic contrast could be detected in remanence by performing a pixel-by-pixel difference of X-ray absorption images acquired with X-rays of opposite circular polarization at a given photon energy. In figure $7 b-d$ we show XMCD images acquired at different photon energies. The images shown in Figure $7 c$ and $d$ are acquired at photon energies of 707.2 and $708 \mathrm{eV}$, which correspond to the two most prominent peaks of the $\mathrm{Fe}$ XMCD spectrum (Figure $5 \mathrm{c}$ ). The same two domains are marked in the different images with yellow arrows. The XMCD image shows a fine structure of roundish magnetic domains which appear white and black, together with interdigitated gray areas. The XMCD contrast is proportional to the component of the magnetization along the $\mathrm{X}$-ray direction, which in the ALBA setup is near in-plane $\left(16^{\circ}\right.$ incidence
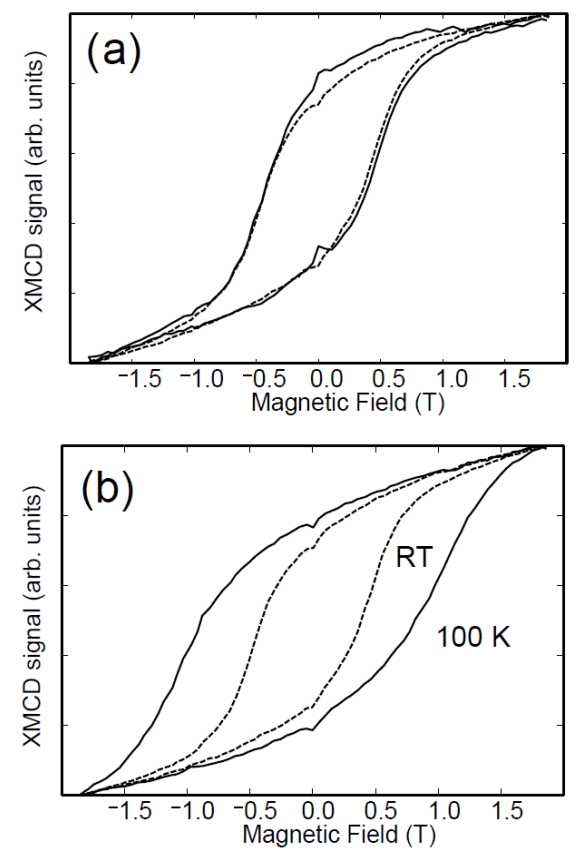

Figure 6. (a) Hysteresis cycles at room temperature acquired at the $\mathrm{Co}(778 \mathrm{eV}$, continuous line) and the $\mathrm{Fe}(708 \mathrm{eV}$, dashed line) XMCD peaks; (b) Hysteresis cycles at the same energy at $R T$ (dashed line) and at $100 \mathrm{~K}$ (continuous like). angle). The magnetic origin of the observed contrast is confirmed by the reversal of the contrast when switching the photon energy. Furthermore, the same magnetic domains are observed at the $\mathrm{Co}_{3}$ peak, indicating that they arise from areas which contain both Fe and Co.

\section{CONCLUSION}

We have characterized polycrystalline targets of cobalt ferrite, and the films grown from them by infrared pulsed laser deposition on $\mathrm{SrTiO}_{3}$ : $\mathrm{Nb}$ substrates. Mössbauer spectroscopy is brought forwards as a crucial technique to disentangle the composition of the films, which are composed of a coherent mixture of a slightly reduced cobalt ferrite and a magnetite phase. The magnetic domains of the films have been imaged by XMCD in photoemission microscopy, and the orbital and spin components of the magnetic moments of iron and cobalt have been estimated by XMCD. We proposed that the route presented here to grow structurally and magnetically coherent mixtures of magnetite and cobalt ferrite constitutes a new way to tune the properties of cobalt-iron ferrites.
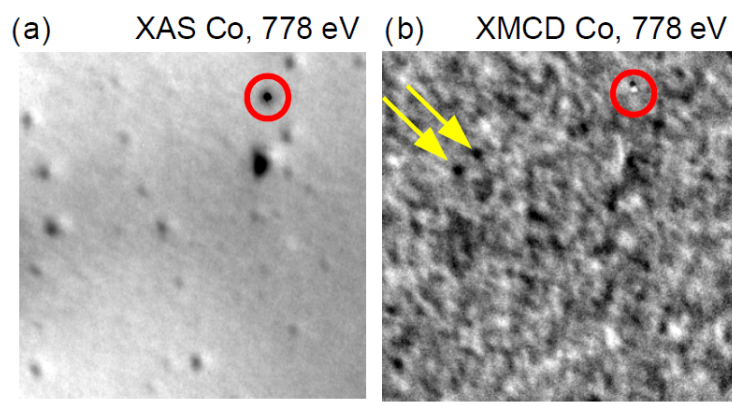

(c) XMCD Fe, $707.2 \mathrm{eV}$
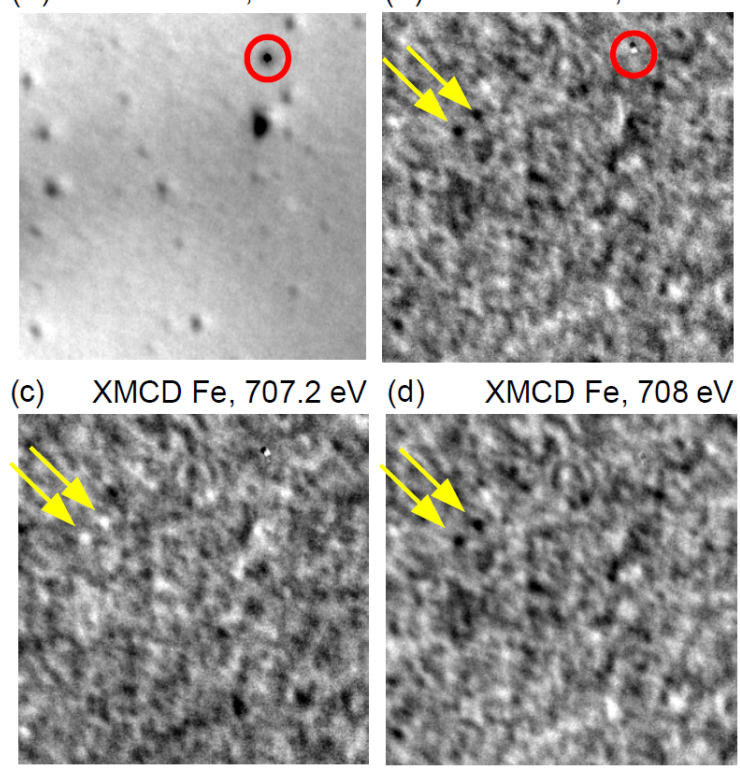

Figure 7. (a) XAS-PEEM image at the peak of the Co $L_{3}$ edge; (b) XMCD-PEEM image of the magnetic domains imaged at the $\mathrm{Co}_{3}$ absorption edge; (c) and (d) XMCD-PEEM image of the magnetic domains imaged at the $\mathrm{Fe}_{3} \mathrm{~L}_{3}$ absorption edge, at two energies where the contrast reverses. The same spot is marked by a red circle in the Co images. The same magnetic domains, $50 \mathrm{~nm}$ wide, are marked with yellow arrows across all the XMCD images. The lateral size of the images is $3.4 \mu \mathrm{m}$. 
Acknowledgment. This research was supported by the Spanish Ministry of Economy and Competitiveness (MINECO) through Projects No. MAT2012-38045-C04-01, CTQ2013-43086-P, and MAT2013-48009-C4-1-P and by the EU-FP7 NANOPYME Project (No. 310516). The PEEM experiments were performed at the CIRCE beamline of the ALBA Synchrotron Light Facility with the collaboration of ALBA staff. The Advanced Light Source is supported by the Director, Office of Science, Office of Basic Energy Sciences, of the U.S. Department of Energy under Contract No. DE-AC02-05CH11231. L.M.-G. acknowledges support from an FPI contract with reference BES-2013-063396 from the MINECO.

\section{REFERENCES}

[1] V. A. Brabers, Handbook of Magnetism and Advanced Magnetic Materials, John Wiley \& Sons, Ltd, 2007.

[2] J. A. Moyer, C. A. F. Vaz, E. Negusse, D. A. Arena, V. E. Henrich, Phys. Rev. B 2011, 83, 035121.

[3] J. A. Moyer, C. A. F. Vaz, D. A. Arena, D. Kumah, E. Negusse, V. E. Henrich, Phys. Rev. B 2011, 84, 054447.

[4] M. Opel, J. Phys. D 2012, 45, 033001.

[5] F. W. Oliver, D. Seifu, E. J. Hoffman, V. Webb, Hyperfine Inter. 2004, 153, 57.

[6] A. V. Ramos, T. S. Santos, G. X. Miao, M.-J. Guittet, J.-B. Moussy, J. S. Moodera, Phys. Rev. B 2008, 78, 180402.

[7] F. Rigato, S. Piano, M. Foerster, F. Giubileo, A. M. Cucolo, J. Fontcuberta, Phys. Rev. B 2010, 81, 174415.

[8] G. Dascalu, O. F. Caltun, J. Optoelectron. Adv. M. 2011, 13, 1145.

[9] M. Monti, M. Sanz, M. Oujja, E. Rebollar, M. Castillejo, F. J. Pedrosa, A. Bollero, J. Camarero, J. L. F. Cuñado, N. M. Nemes, F. J. Mompean, M. GarciaHernández, S. Nie, K. F. McCarty, A. T. N’Diaye, G. Chen, A. K. Schmid, J. F. Marco, J. de la Figuera, J. App. Phys. 2013, 114, 223902.

[10] M. Sanz, M. Oujja, E. Rebollar, J. F. Marco, J. de la Figuera, M. Monti, A. Bollero, J. Camarero, F. J. Pedrosa, M. García-Hernández, M. Castillejo, Appl. Surf. Sci. 2013, 282, 642.
[11] J. R. Gancedo, M. Gracia, J. F. Marco, Hyperfine Interact. 1991, 66, 83.

[12] J. R. Gancedo, M. Gracia, J. F. Marco, Hyperfine Interact. 1994, 83, 71.

[13] A. Locatelli, L. Aballe, T. O. Mentes, M. Kiskinova, E. Bauer, Surf. Interface Anal. 2006,38, 1554.

[14] L. Aballe, M. Foerster, E. Pellegrin, J. Nicolas, S. Ferrer, J. Synchrotron Rad. 2015, 22, 745.

[15] Kevin F. McCarty, Juan de la Figuera, Surface Science Techniques, Springer Series in Surface Sciences, Vol. 51, Springer Berlin Heidelberg, 2013, p. 531.

[16] S. J. Kim, S. W. Lee, C. S. Kim, Jap. J. Appl. Phys. 2001, 40, 4897.

[17] M. Sorescu, A. Grabias, R. A. Brand, J. Voss, D. Tarabasanu-Mihaila, L. Diamandescu, J. Mag. Mag. Mat. 2002, 246, 399.

[18] M. F. F. Lelis, A. O. Porto, C. M. Gonalves, J. D. Fabris, J. Mag. Mag. Mat. 2004, 278, 263.

[19] A. Quesada, F. Rubio-Marcos, J. F. Marco, F. J. Mompean, M. García-Hernández, and J. F. Fernández, Appl. Phys. Lett. 2014, 105, 202405.

[20] M. Takahashi, M. E. Fine, J. App. Phys. 1972, 43, 4205.

[21] R. E. Vandenberghe, E. D. Grave, in Modern Inorganic Chemistry, No. 3 (Eds.: G. J. Long, F. Grandjean), Springer US, 1989, pp. 59-182.

[22] S. Jin Kim, S. Wha Lee, S. Yong An, C. S. Kim, J. Mag. Magn. Mat. 2000, 210, 215.

[23] J. de la Figuera, A. Quesada, L. Martín-García, M. Sanz, M. Oujja, E. Rebollar, M. Castillejo, P. Prieto, A. M. noz Martín, L. Aballe, J. F. Marco, App. Surf. Sci. 2015, 359, 480.

[24] E. Pellegrain, M. Hagelstein, S. Doyle, H. Moser, J. Fuchs, D. Vollath, S. Schuppler, M. James, S. Saxena, L. Niesen, O. Rogojanu, G. Sawatzky, C. Ferrero, M. Borowski, O. Tjernberg, N. Brookes, Phys. Stat. Sol. B 1999, 215, 797.

[25] C. T. Chen, Y. U. Idzerda, H.-J. Lin, N. V. Smith, G. Meigs, E. Chaban, G. H. Ho, E. Pellegrin, F. Sette, Phys. Rev. Lett. 1995, 75, 152.

[26] C. M. Schneider, G. Schönhense, Rep. Prog. Phys. 2002, 65, 1785. 